\title{
Surface-enhanced Raman spectroscopy: nonlocal limitations
}

Toscano, Giuseppe; Raza, Søren; Xiao, Sanshui ; Wubs, Martijn; Jauho, Antti-Pekka; Bozhevolnyi, Sergey I.; Mortensen, N. Asger

\section{Published in:}

Optics Letters

Link to article, DOI:

10.1364/OL.37.002538

Publication date:

2012

Document Version

Publisher's PDF, also known as Version of record

Link back to DTU Orbit

Citation (APA):

Toscano, G., Raza, S., Xiao, S., Wubs, M., Jauho, A-P., Bozhevolnyi, S. I., \& Mortensen, N. A. (2012). Surfaceenhanced Raman spectroscopy: nonlocal limitations. Optics Letters, 37(13), 2538-2540.

https://doi.org/10.1364/OL.37.002538

\section{General rights}

Copyright and moral rights for the publications made accessible in the public portal are retained by the authors and/or other copyright owners and it is a condition of accessing publications that users recognise and abide by the legal requirements associated with these rights.

- Users may download and print one copy of any publication from the public portal for the purpose of private study or research.

- You may not further distribute the material or use it for any profit-making activity or commercial gain

- You may freely distribute the URL identifying the publication in the public portal 


\title{
Surface-enhanced Raman spectroscopy: nonlocal limitations
}

\author{
G. Toscano, ${ }^{1}$ S. Raza,${ }^{1,2}$ S. Xiao, ${ }^{1}$ M. Wubs, ${ }^{1}$ A.-P. Jauho, ${ }^{3}$ S. I. Bozhevolnyi,${ }^{4}$ and N. A. Mortensen ${ }^{1, *}$ \\ ${ }^{1}$ Department of Photonics Engineering, Technical University of Denmark, DK-280o Kgs. Lyngby, Denmark \\ ${ }^{2}$ Center for Electron Nanoscopy, Technical University of Denmark, DK-2800 Kgs. Lyngby, Denmark \\ ${ }^{3}$ Department of Micro and Nanotechnology, Technical University of Denmark, DK-2800 Kgs. Lyngby, Denmark \\ ${ }^{4}$ Institute of Sensors, Signals and Electrotechnics, University of Southern Denmark, DK-5230 Odense, Denmark \\ *Corresponding author: asger@mailaps.org
}

Received March 8, 2012; revised May 1, 2012; accepted May 10, 2012;

posted May 11, 2012 (Doc. ID 164355); published June 21, 2012

\begin{abstract}
Giant field enhancement and field singularities are a natural consequence of the commonly employed localresponse framework. We show that a more general nonlocal treatment of the plasmonic response leads to new and possibly fundamental limitations on field enhancement with important consequences for our understanding of surface-enhanced Raman spectroscopy (SERS). The intrinsic length scale of the electron gas serves to smear out assumed field singularities, leaving the SERS enhancement factor finite, even for geometries with infinitely sharp features. For silver nanogroove structures, mimicked by periodic arrays of half-cylinders (up to $120 \mathrm{~nm}$ in radius), we find no enhancement factors exceeding 10 orders of magnitude $\left(10^{10}\right)$. @ 2012 Optical Society of America

OCIS codes: $240.6680,290.5860,300.6450$.
\end{abstract}

While the Raman response of (bio)molecules is inherently weak, nanostructures may be used to tailor and tremendously enhance the light-matter interactions. This is the key electromagnetic element of surface-enhanced Raman spectroscopy (SERS) [1]. In particular, metallic nanostructures [2] are known to support plasmonic field-enhancement phenomena that are beneficial for SERS [3]. In many cases, field singularities arise in geometries with abrupt changes in the surface topography. While such singularities constitute the basic electromagnetic mechanism behind SERS, the singularities are, on the other hand, an inherent consequence of the common localresponse approximation (LRA) of the plasmons [4]. In this Letter, we relax this approximation and allow for nonlocal dynamics of the plasmons. To illustrate the consequences, we revisit the model geometry in Fig. 1 , initially put forward by García-Vidal and Pendry [ㅁ] to qualitatively explain the electromagnetic origin of the large enhancement factors observed experimentally. The metallic surface topography is composed of a periodic structure of infinitely long metallic half-cylinders of radius $R$, resting shoulder-by-shoulder on a semiinfinite metal film. The steep trenches or grooves support localized surface plasmon resonances (LSPRs). Near the bottom of the groove the surfaces of the two touching half-cylinders become tangential to each other and a field singularity forms within the traditional LRA of the dielectric function. In the common treatment, the field enhancement thus eventually turns infinite []], while it remains finite, albeit large, in any experiment reported so far. Geometrical smoothening is known to remove the singularity within the LRA and, in quantitative numerical studies, a rounding needs to be added to make numerical convergence feasible $[\underline{7}, 8]$. Thus, within the LRA framework the field enhancement would just grow without bound the sharper one could make the geometry confining the plasmon oscillations. Nonlocal effects have been shown to result in large blueshifts and considerably reduced field enhancements (as compared to a local description) in conical tips [9] , metallic dimers involving small gaps below a few nanometers [10,11], or even vanishing gaps [12]. What is the limit in field enhancements that can be achieved with (geometrically) ideal structures? This question is important not only from the fundamental but also from the applied perspective, as the answer to it would allow one to determine technological tolerances in fabrication of nanostructures designed for achieving record-high field enhancements. In this Letter we show how nonlocal response introduces a new intrinsic length scale that serves to remove the field singularities, leaving field enhancements finite even in geometries with arbitrarily sharp changes in the surface topography. For the particular geometry of Fig. 1 we evaluate $\gamma(\mathbf{r}, \omega)=|\mathbf{E}(\mathbf{r}, \omega)|^{4} /\left|\mathbf{E}_{0}(\omega)\right|^{4}$ and find no (surface-averaged) SERS enhancementfactors $\langle\gamma\rangle$ exceeding 10 orders of magnitude.

The electromagnetic response of a metal is commonly divided into intraband contributions [13] and the dispersive Drude free-electron response

$$
\varepsilon_{D}(\omega)=1+i \frac{\sigma}{\varepsilon_{0} \omega}=1-\frac{\omega_{p}^{2}}{\omega\left(\omega+i / \tau_{D}\right)}
$$
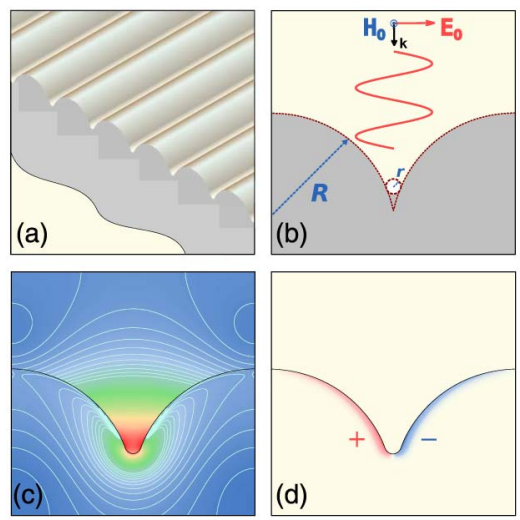

Fig. 1. (Color online) (a) Groove structure formed by an infinite periodic array of half-cylindrical nanorods. (b) Cross section of the unit cell. (c) and (d) Typical electric-field intensity and charge distributions for a dipole mode. 
where $\sigma$ is the complex conductivity also appearing in Ohm's law $\mathbf{J}=\sigma \mathbf{E}$. We relax the latter local-response constitutive equation and turn to a linearized hydrodynamic nonlocal treatment $[10,11,14,15]$ where the usual Maxwell wave equation is coupled to a hydrodynamic equation for the current density; see [11] for the full details of our numerical approach. This is the simplest nontrivial extension of the common LRA Drude model, which, in addition to the usual metal parameters $\left(\omega_{p}\right.$, $\tau_{D}$, etc.), now also carries information about the kinetics of the charge carriers at the Fermi level. The strength of the nonlocal correction to Ohm's law depends on the Fermi velocity $v_{F}$, which introduces a new length scale, being a factor $v_{F} / c$ of the free-space wavelength $\lambda=2 \pi c / \omega$. For the noble metals, $v_{F} / c$ is of the order $10^{-2}$, which explains the overall success of the LRA. However, when exploiting plasmonics at the true nanoscale, effects due to the nonlocal dynamics start to manifest themselves. Field-enhancement structures turn out to be prime examples of this.

We consider the metallic groove structure shown in Fig. 1, which has previously been considered as a model system to mimic corrugated metal surfaces [5]. Alternatively, it may be viewed as a model for arrays of the more recent groove or channel waveguides $[\underline{7,16}]$. In our numerical study, the structure is excited by an incoming plane wave $\mathbf{E}_{0}(\omega)$, normal to the substrate and with the field polarized perpendicularly to the axis of the halfcylinders, i.e., across the groove cross section. Noble metals are common choices for plasmonics and in the following we focus our attention on silver [13]. The grooves have been shown to support LSPRs [7]], which we have previously explored in the context of SERS, using a LRA and with the necessary addition of geometrical smoothening [8]. To quantify the SERS effect and the consequences of nanoscale spatial dispersion, we solve the nonlocal wave equation numerically [11]. As an example of our results, Fig. 2 shows the spectral dependence of $\langle\gamma\rangle$ throughout the visible regime for groove structures with $R=75 \mathrm{~nm}$ and with a radius of curvature of the crevice given by $r=0.1 \mathrm{~nm}$. The LSPR at $\lambda=$ $700 \mathrm{~nm}$ allows the (surface-averaged) Raman rate to

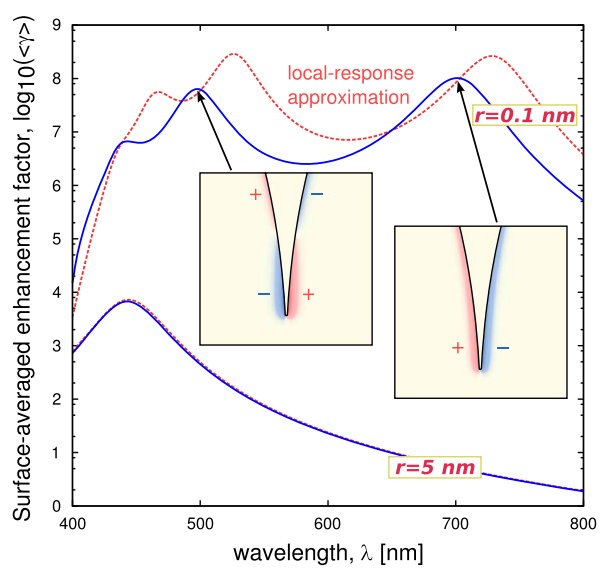

Fig. 2. (Color online) Surface-averaged SERS enhancement factor $\langle\gamma\rangle$ for the case of $R=75 \mathrm{~nm}$ with $r=0.1 \mathrm{~nm}$ (upper curves) and $r=5 \mathrm{~nm}$ (lower curves). For comparison, the dashed curves show the results of the commonly employed LRA. be enhanced by a factor of $10^{8}$. For comparison, the dashed curve shows results when treating the plasmonic response within the common LRA. In both cases, the resonant behavior is well pronounced, being caused by interference of the incoming field with the gap surface plasmon mode reflected at the bottom, similarly to that described for $\mathrm{V}$ grooves [16]. As a general fingerprint of nonlocal response, the peak is blueshifted compared to the expectations from a local-response treatment of the problem (this happens due to a decrease in the gap plasmon index caused by nonlocal effects [10]). In this particular case, the LSPR by the common treatment is off by more than $25 \mathrm{~nm}$, which illustrates the importance of nonlocal effects for quantitative SERS predictions. Even more importantly, the common LRA is seen to significantly overestimate the enhancement factor; for some wavelengths by more than 1 order of magnitude. The large quantitative differences between the nonlocal treatment and the traditional LRA are associated with changes in the induced-charge distribution (insets of Fig. 2). In the common treatment, the charge is strictly a surface charge, while in the general nonlocal case the intrinsic scale $v_{F} / \omega$ serves to spatially smear out the charge distribution. Effectively, this smearing increases the electric-field penetration into the metal (silver) and thereby increases the field absorption (ohmic loss) and damping of resonant oscillations. Interpreting the field enhancement in a capacitor picture, the finite thickness of the charge distribution near the surface increases the effective separation (beyond that given by the metalsurface geometry) and, consequently, the capacitor supports a lower electrical field compared to in the LRA. In general, the intrinsic length scale of the electron gas allows one to resolve the field also in the proximity of very sharp corners and tips. On the other hand, by relaxing the sharpness of the trench the influence of spatial dispersion becomes less pronounced, as illustrated in Fig. 2 in the lower set of curves $(r=5 \mathrm{~nm})$, where the LRA accounts well for the results obtained from a full nonlocal treatment. We note a drastic change in the fieldenhancement spectrum, with the fundamental resonance now appearing at around $450 \mathrm{~nm}$, due to a very rapid decrease in the gap plasmon index when the gap width increases (at the groove bottom) from 0.1 to $5 \mathrm{~nm}$.

With less geometrical smoothening (i.e., when $r$ is made smaller and smaller), the shortcomings of the LRA become more severe. The LRA anticipates a monotonically increasing enhancement factor []ㅡ, and decreasing $r$ also causes a stronger interaction between neighboring half-cylinders and, consequently, a redshift [5]. Note that, in the interpretation based on gap surface plasmons [16], the redshift is simply related to an increase in the gap plasmon index when the gap width decreases at the groove bottom. In Fig. 3 we decrease $r$ from $1 \mathrm{~nm}$ down to zero and see how nonlocal effects cause a different trend (indicated by the dashed curve) due to the competing length scales. In particular, for $r \lesssim v_{F} / \omega$, there is a fundamental saturation of the enhancement factor rather than a monotonic increase and, for our particular choice of the cylinder radius $R$, we see that the $\langle\gamma\rangle$ does not exceed $2 \times 10^{9}$.

To explore the ultimate limitations on the SERS in this geometry, Fig. $\underline{4}$ shows results where we have completely 


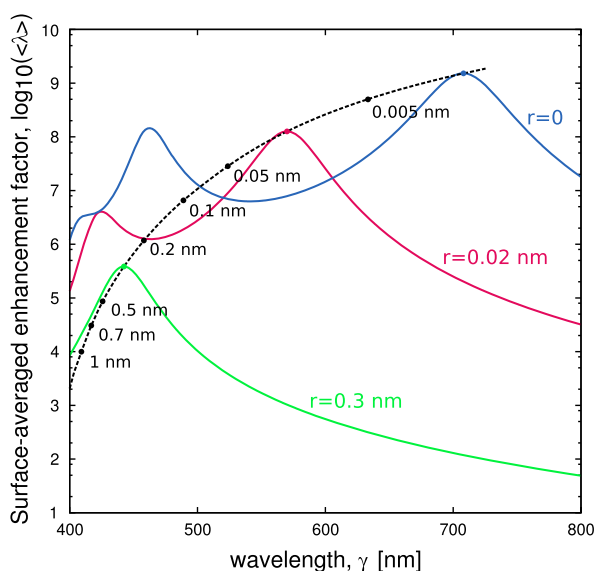

Fig. 3. (Color online) Surface-averaged SERS enhancement factor $\langle\gamma\rangle$ for the case of $R=15 \mathrm{~nm}$ and with $r$ varying from 1 to $0 \mathrm{~nm}$. The dashed curve connecting fundamental dipole resonances for different values of $r$ serves as a guide to the eyes, clearly illustrating both a redshift and the saturation effect in the field enhancement as $r \rightarrow 0$.

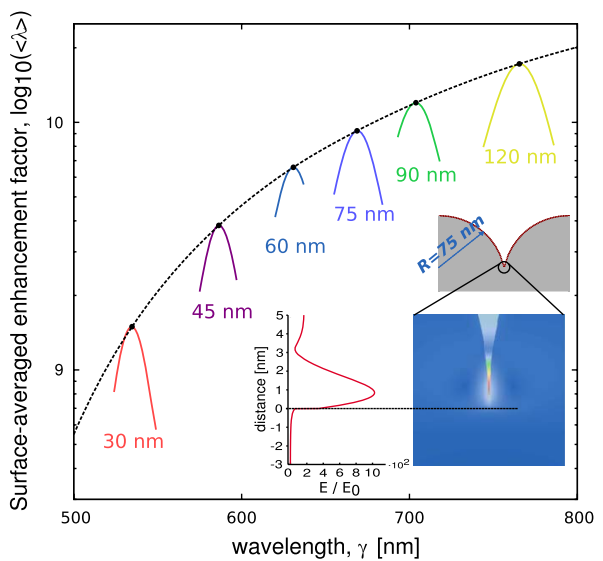

Fig. 4. (Color online) Near-resonance plots of the surfaceaveraged SERS enhancement factor $\langle\gamma\rangle$ for arbitrarily welldefined grooves without smoothening $(r=0)$ for six cases with $R$ varying from 30 to $120 \mathrm{~nm}$. The inset shows the field-amplitude distribution $|\mathbf{E}| /\left|\mathbf{E}_{0}\right|$ for $R=75 \mathrm{~nm}$.

refrained from any geometrical smoothening $(r=0)$ and where $v_{F} / \omega$ is the only length scale that puts fundamental limitations on the field enhancement. As the radius $R$ of the half-cylinders is increased from 30 to $120 \mathrm{~nm}$ we see a redshift of the peak, as also anticipated in the LRA [8]. At the same time, the enhancement factor exhibits an increasing trend where larger cylinders support larger field enhancement by harvesting the incoming field from larger areas. We emphasize that in all examples the field enhancement remains finite despite the fact that the crevice is arbitrarily sharp and well defined $(r=0)$. For the largest radius $R$ considered the electromagnetic SERS enhancement factor does not exceed $2 \times 10^{10}$. This illustrates the fundamental limitations imposed by nonlocal response in our specific SERS configuration.

In conclusion, we have shown that a nonlocal treatment of the plasmonic response leads to new and possibly fundamental limitations on the electromagnetic SERS enhancement factor, thereby completely changing the message of the commonly employed LRA of the plasmons. The intrinsic length scale of the electron gas serves to smear out the field singularity that otherwise would arise from a local-response treatment and, as a consequence, the enhancement remains finite even for geometries with infinitely sharp features. Finally, beyond the linear response, fundamental limitations may arise due to nonlinearities [17].

\section{References}

1. M. Moskovits, Rev. Mod. Phys. 57, 783 (1985).

2. S. Lal, S. Link, and N. J. Halas, Nat. Photon. 1, 641 (2007).

3. K. Kneipp, Phys. Today 60(11), 40 (2007).

4. Y. Luo, J. B. Pendry, and A. Aubry, Nano Lett. 10, 4186 (2010).

5. F. García-Vidal and J. B. Pendry, Phys. Rev. Lett. 77, 1163 (1996)

6. I. Romero, J. Aizpurua, G. W. Bryant, and F. J. García de Abajo, Opt. Express 14, 9988 (2006).

7. E. Moreno, F. J. García-Vidal, S. G. Rodrigo, L. MartínMoreno, and S. I. Bozhevolnyi, Opt. Lett. 31, 3447 (2006).

8. S. Xiao, N. A. Mortensen, and A.-P. Jauho, J. Eur. Opt. Soc. Rapid Pub. 3, 08022 (2008).

9. R. Ruppin, Phys. Lett. A 340, 299 (2005).

10. F. J. García de Abajo, J. Phys. Chem. C 112, 17983 (2008).

11. G. Toscano, S. Raza, A.-P. Jauho, N. A. Mortensen, and M. Wubs, Opt. Express 20, 4176 (2012).

12. A. I. Fernández-Domínguez, A. Wiener, F. J. García-Vidal, S. A. Maier, and J. B. Pendry, Phys. Rev. Lett. 108, 106802 (2012).

13. S. G. Rodrigo, F. García-Vidal, and Martín-Moreno, Phys. Rev. B 77, 075401 (2008).

14. J. M. Pitarke, V. M. Silkin, E. V. Chulkov, and P. M. Echenique, Rep. Prog. Phys. 70, 1 (2007).

15. S. Raza, G. Toscano, A.-P. Jauho, M. Wubs, and N. A. Mortensen, Phys. Rev. B 84, 121412(R) (2011).

16. T. Søndergaard, S. I. Bozhevolnyi, J. Beermann, S. M. Novikov, E. Devaux, and T. W. Ebbesen, Nano Lett. 10, 291 (2010).

17. P. Ginzburg, A. Hayat, N. Berkovitch, and M. Orenstein, Opt. Lett. 35, 1551 (2010). 